\title{
A simple and rapid diagnostic method for 13 types of High risk human papillomavirus (HR-HPVs) detection using CRISPR-Cas12a technology
}

Jiaojiao Gong

Yaneng Biotech, Co., Ltd, Fosun Pharma

Guanghui Zhang

Shen zhen hengsheng hospital

Wangguo Wang

the Second People's Hospital of Shangrao

Liping Liang

Shen zhen hengsheng hospital

Qianyun Li

University of Chinese Academy of Sciences

Menghao Liu

Yaneng Biotech, Co., Ltd, Fosun Pharma

Liang Xue ( $\nabla$ xuel0620@sina.com )

Yaneng Biotech, Co., Ltd, Fosun Pharma

Guanghui Tang ( $\nabla$ tangguanghui@yanengbio.com )

Yaneng Biotech, Co., Ltd, Fosun Pharma

\section{Research Article}

Keywords: HR-HPVs, CRISPR-Cas12a, non-genotyping

Posted Date: April 13th, 2021

DOl: https://doi.org/10.21203/rs.3.rs-396579/v1

License: (1) (1) This work is licensed under a Creative Commons Attribution 4.0 International License.

Read Full License 


\section{Abstract}

Cervical carcinoma is the second most common cancer in women worldwide with greater than $99 \%$ of the cases caused by human papillomaviruses (HPVs). Early detection of HPVs especially the high risk types (HR-HPVs) are essential to prevent the disease progression. The existing methods for HPV detection, such as qPCR are of high sensitivity and specificity, but the need for expensive machinery and well-trained personnel slow down the disease detection. The emerging Cas12a-based method presents a new technique for nucleic acid detection. However, it is time-consuming and labor-intensive when used for HPV detection, as several reactions are required in order to identify multiple HPV infections. We herein present a non-genotyping method for 13 types of HR-HPV detection in a single reaction by combining the isothermal recombinase polymerase amplification (RPA) method with CRISPR-Cas12a technology. The result could be achieved in 30 minutes with high sensitivity (500 copies per reaction). This assay reprsents great advances for the application of RPA-Cas12a system and holds a great potential to address the key challenges facing the HPV diagnostics.

\section{Introduction}

Human papillomavirus (HPV) infection is a major causative agent of cervical cancer in women[1]. In 2018, approximately 311000 women died from cervical cancer and more than $85 \%$ of these deaths occurred in low- and middle-income countries[2]. HPV types can be classified into high risk (HR) and low risk (LR). The HR types (e.g., types 16, 18, 31, 33, 35, 39, 45, 51, 52, 56, 58, 59, 68) are known to be closely associated with preneoplastic lesions and carcinomas, while the LR types (e.g., types 6, 11, 40, 42, 43, 44, $54,61,70,72,81)$ tend to cause warts[3]. Early detection of the HR-HPVs using a simple and rapid method is extremely important for therapeutic action against cervical cancer particularly in areas where specialized equipment is not available[4].

To date, a variety of primer combinations amplifying different regions of the HPV genome have been developed using quantitative polymerase chain reaction (qPCR) technology. The MY09/MY11 primer set and the GP5+/GP6 + primer set, both targeting the conserved L1 region, are the most frequently used amplification systems for the detection of HPV DNA in clinical samples[5-7]. The former of which includes several degenerate nucleotides in each primer while the latter consists of a fixed nucleotide sequence for each primer, and both of them have shown capability of amplifying a wide spectrum of HPV types[8].

The qPCR technology, although known as an effective tool for detection and typing of viruses, is faced with disadvantages such as complicated procedures and requirement of expensive machinery $[9,10]$. The emerging isothermal amplification technologies, recombinase polymerase amplification (RPA) and loopmediated isothermal amplification (LAMP) for examples, have been employed to diagnose HPVs[3, 11]. These methods do not rely on thermal cycling and therefore are suitable for resource-restricted areas. However, they are time-consuming and labor-intensive when genotyping specimens containing multiple 
HPV types, as only one type of HPV could be detected in a given reaction. In some cases, it might not be necessary to genotype all individual HPV types, but rather detecting all HR-HPVs as a whole.

The clustered regularly interspaced short palindromic repeats (CRISPR) and Cas (CRISPR associated proteins) are adaptive immune systems in archaea and bacteria[12, 13]. Some Cas nucleases display strong collateral activities after binding to their specific cis targets, which has been fully evaluated for diagnostic use[12, 14]. After RPA amplification, Cas12a has been shown to permit a single molecule detection in a given reaction $[15,16]$.

In current study, we present a non-genotyping approach for 13 types of HR-HPVs detection using RPACas12a system, with which the infection status of an individual by HR-HPVs could be clearly identified. Our assay consists of an RPA amplification using the primer pool derived from PGMY/GP6 + primer set, and followed by Cas12a-mediated detection (Fig. 1). To our knowledge, this is the first report realizing mutiple HPV testing in a singal reaction with the RPA-Cas system. Hence, our research represents significant advances for the application of this system. Because the present assay is fast and needs minimal equipment, its application may help the triage procedure of the cervical cancer screening.

\section{Results And Discussion}

According to the guidelines for nucleic acid testing of HPV released by Chinese Food and Drug Administration (CFDA) in 2015, 13 types of HR-HPV (types 16, 18, 31, 33, 35, 39, 45, 51, 52, 56, 58, 59, 68) are required to be identifed when developing a diagnostic assay. We therefore designed a pool that inculdes 13 pairs of RPA primers targeting the L1 conservative region of the HR-HPVs (Table S1). To ensure a rapid and sensitive RPA reaction, all primers were synthesized at the length between 30 and 36 oligonucleotides. The kinetic fluorescence curve of Cas12a reaction showed that only a few of HPV types generated strong signal, indicating weak amplification for most HPV types with this primer pool (Figure S1). This could be due to the production of primer dimers, which consumed the materials in the reaction, and whereby reducing the intensity of specific amplification. We hypothesized that shorter and degenerated primers may reduce the complexity of the primer pool, and subsequently increase the amplification. The commonly used PCR primer sets meet such qualifications, but it is still unknown whether they are compatible with the RPA system. As a proof-of-concept assay, we first evaluated the SPF1A, SPF1B and SPF2D primer combination (Fig. 2A, Table S2) in which the inosine was included to reduce the mismatches to targets[17]. Howerver, no signal was observed when genomic DNA from siHA cell that is positive for HPV16 was tested (Figure S2). We next evaluated another commonly used primer set GP5/GP6 for 6 types of HR-HPV detection. Although this primer set was able to amplify HPV16, HPV18 and HPV31, it falled to detect HPV33, HPV35 and HPV39, suggesting it was not a perferct candidate for all 13 types of HR-HPV detection (Figure S3).

Then, we applied the PGMY/GP6 + primer pool to detect plasmids of HPV16 and HPV18 (Fig. 2A). As shown in Fig. 2B, this pool could detect both types with high sensitivity (100 copies for a single reaction). All crRNAs targeting the sequences flanked by PGMY/GP6 + were designed and validated with $5 \mu \mathrm{g}$ of 
each individual HPV plasmid (Fig. 2C). The primer pool was further tested for the rest of HR-HPVs using 10000 copies for RPA amplification in a reaction. The result demonstrated that most types was able to be efficiently amplified except HPV31, 39, 51, 56, 59, 68 (Fig. 2D). After aligned the GP6 + sequence to genomic sequence of these six HPV types, we found significant mismathes that may result in the failure of amplification (Fig. 2E). To expand the spectrum of this primer pool for other HR-HPVs, we supplemented additional four primers that has better matches to these six HPV types into the pool. With the new pool, all the 13 types of HR-HPVs were successfully detected when 10000 copies of plasmid for each RPA reaction was used (Fig. 3).

The sensitivity of our assay was subsequently analyzed, and the results showd that it was able to detect all HPV types in a reaction containing 500 copies of plasmid (Fig. 4). A much higher sensitivity to HPV16, $18,31,35,52$, and 68 was achieved, where the limit of detection (LoD) was 100 copies for a given reaction (Fig. 4). Our assay seems not to be as senstive as the reported ones in which the LoD of single molecule was observed[15, 18]. The multiple RPA amplification could be the reason leading to the limited sensitivity of the assay, which is consistent with the research by Zachary et. al[19]. Besides, the use of shorter primers could be another reason for the higher LoD in our assay, as shorter primers would be less efficient in amplifiying the targets. The final optimized primer pool includes a total of ten primers (5 PGMY11 and 5 GP6+, Table S3), and the crRNA pool consists of 11 crRNAs, with one crRNA recognizing three HPV types (HPV18, 31, 33, Table S4). According to the instruction described in the protocol of TwistDx, the total amount of oligonucleotides in the reaction mixture should not exceed $1000 \mathrm{nM}$. However, the concentration of primers in current study $(4000 \mathrm{nM}$ in total) was far more than the standard, we therefore titrated the primers in reactions for detection of HPV16. The result showed that $4000 \mathrm{nM}$ of primers in a reaction generated the strongest signal, indicating the highest amplification efficiency at this concentration (Figure S4).

We next compared the sensitivity of the RPA-Cas12a-based method with the RPA-only method by detection of plasmid of HPV16. The RPA-only appoach demonstrated an inferior sensitivity than RAPCas12a-based assay with LoD of $10^{4}$ copies in a reaction (Figure S5). The performance of only Cas12a detection for HPV16 without RPA amplification was also assessed, and the results showed it could detect as low as $0.1 \mu \mathrm{g}$ (equivalent to around $10^{9}$ copies) of HPV16 plasmids in a reaction. However, when the amont of plasmid decreased to $0.01 \mu \mathrm{g}$ and $0.001 \mu \mathrm{g}$ per reaction, there was almost no signal detected (Figure S6). These results together illustrate the necessity of combining RPA amplification with Cas12a technology.

To interrogate any potential false positive, the specificity of our assay to 8 other HPV types and 8 ohter pathogens commonly existed in vagina was evaluated. As shown in Fig. 5, cross-reactivity was not observed with HR-HPVs and the tested pathogens, indicating high specificity of the assay.

Finally, the clinical performance of the system was validated by analysis of materials from cervical scraps. All of the positive samples previously identifed by qPCR were positive in our assay, and all the qPCR-negative samples were negative by our assay for detection of the 13 types of HPVs (Fig. 6). 
Although the developed assay had $100 \%$ positive and negative agreements relative to the qPCR assay, the results should be carefully interpreted due to the limited number of specimens for each type. A larger cohort would be helpful to better understand the assay's sensitivity and specificity in future studies.

In summary, we report the development of an RPA-Cas12a-based non-genotyping method for detection of 13 types of HR-HPVs, which could serve as an useful tool for screening and triage of cervical cancer. Our study generally provides an easy approach for HR-HPV testing, however, this non-genotyping method may encounter substantial limitations since it is not able to genotype the most carcinogenic HPV types HPV16 and HPV18. To overcome this, additional reactions with primer sets of PGMY11-B and GP6 + for HPV16 and PGMY11-A and GP6 + for HPV18 could be performed (data not shown). Moreover, our method needs a tube-opening operation to transfer the RPA reaction for Cas12a cleavage, and this could generate aerosol and cause false-positive results. To avoid amplicon contamination, the tube opening should be strictly performed in a separate area. An alternative strategy was to add the Cas enzyme on the inner wall of the tube, and followed by a centrifugation step to start the Cas12a detection after RPA amplification[20].

Our work also provides a novel concept to use RPA-based nucleic acid amplification. Previous studies has realized triplex detections using the RPA technology even though the sensitivity was significantly reduced compared with the signleplex detection[19]. Nevertheless, when facing with more pathogen types, it is still a great challenge to apply the isothermal amplification-based methods for nucleic acid detection, particularly when using the RPA method. This might be due to the generation of primer dimers triggered by the second structure of primers as a result of too many primers in a single reaction. In present study, we created a primer pool and a crRNA pool that are capable of detecting 13 types of HR-HPVs in a reaction. The successful application of our method might be explained by the usage of the degenerated PCR primers that are shorter and less complex compared to the commonly used RPA primers. Thus, this novel methodology can be expanded for detection of multiple infections of other pathogens besides HPVs.

\section{References}

1. Bosch FX, Lorincz A, Muñoz N, Meijer C, Shah KVJJocp. The causal relation between human papillomavirus and cervical cancer. 2002;55(4):244-65.

2. Ginsburg O, Bray F, Coleman MP, Vanderpuye V, Eniu A, Kotha SR, et al. The global burden of women's cancers: a grand challenge in global health. 2017;389(10071):847-60.

3. Ma B, Fang J, Lin W, Yu X, Sun C, Zhang M. A simple and efficient method for potential point-of-care diagnosis of human papillomavirus genotypes: combination of isothermal recombinase polymerase amplification with lateral flow dipstick and reverse dot blot. Anal Bioanal Chem. 2019;411(28):745160. Epub 2019/10/08. doi: 10.1007/s00216-019-02113-5. PubMed PMID: 31588523.

4. Urquiza M, Guevara T, Espejo F, Bravo MM, Rivera Z, Patarroyo ME. Two L1-peptides are excellent tools for serological detection of HPV-associated cervical carcinoma lesions. Biochem Biophys Res 
Commun. 2005;332(1):224-32. Epub 2005/05/18. doi: 10.1016/j.bbrc.2005.04.115. PubMed PMID: 15896321.

5. Hildesheim A, Schiffman MH, Gravitt PE, Glass AG, Greer CE, Zhang T, et al. Persistence of typespecific human papillomavirus infection among cytologically normal women. 1994;169(2):235-40.

6. Manos MJCc. The use of polymerase chain reaction amplification for the detection of genital human papillomavirus. 1989;7:209-14.

7. de Roda Husman A-M, Walboomers JM, van den Brule AJ, Meijer CJ, Snijders PJJJogv. The use of general primers GP5 and GP6 elongated at their 3' ends with adjacent highly conserved sequences improves human papillomavirus detection by PCR. 1995;76(4):1057-62.

8. Qu W, Jiang G, Cruz Y, Chang CJ, Ho G, Klein RS, et al. PCR detection of human papillomavirus: comparison between MY09/MY11 and GP5+/GP6+ primer systems. 1997;35(6):1304-10.

9. Jiang HL, Zhu HH, Zhou LF, Chen F, Chen Z. Genotyping of human papillomavirus in cervical lesions by L1 consensus PCR and the Luminex xMAP system. J Med Microbiol. 2006;55(Pt 6):715-20. Epub 2006/05/12. doi: 10.1099/jmm.0.46493-0. PubMed PMID: 16687589.

10. Lee HP, Cho W, Bae JM, Shin JY, Shin SK, Hwang SY, et al. Comparison of the clinical performance of restriction fragment mass polymorphism (RFMP) and Roche linear array HPV test assays for HPV detection and genotyping. J Clin Virol. 2013;57(2):130-5. Epub 2013/02/16. doi:

10.1016/j.jcv.2013.01.014. PubMed PMID: 23410688.

11. Satoh T, Matsumoto K, Fujii T, Sato O, Gemma N, Onuki M, et al. Rapid genotyping of carcinogenic human papillomavirus by loop-mediated isothermal amplification using a new automated DNA test (Clinichip HPV). J Virol Methods. 2013;188(1-2):83-93. Epub 2012/12/12. doi:

10.1016/j.jviromet.2012.10.014. PubMed PMID: 23219807.

12. Chen JS, Ma E, Harrington LB, Da Costa M, Tian X, Palefsky JM, et al. CRISPR-Cas12a target binding unleashes indiscriminate single-stranded DNase activity. Science. 2018;360(6387):436-9. Epub 2018/02/17. doi: 10.1126/science.aar6245. PubMed PMID: 29449511; PubMed Central PMCID: PMCPMC6628903.

13. Barrangou R, Fremaux C, Deveau H, Richards M, Boyaval P, Moineau S, et al. CRISPR provides acquired resistance against viruses in prokaryotes. Science. 2007;315(5819):1709-12.

14. Fozouni P, Son S, Diaz de Leon Derby M, Knott GJ, Gray CN, D'Ambrosio MV, et al. Amplification-free detection of SARS-CoV-2 with CRISPR-Cas13a and mobile phone microscopy. Cell. 2021;184(2):32333 e9. Epub 2020/12/12. doi: 10.1016/j.cell.2020.12.001. PubMed PMID: 33306959; PubMed Central PMCID: PMCPMC7834310.

15. Xiong D, Dai W, Gong J, Li G, Liu N, Wu W, et al. Rapid detection of SARS-CoV-2 with CRISPR-Cas12a. PLoS Biol. 2020;18(12):e3000978. Epub 2020/12/16. doi: 10.1371/journal.pbio.3000978. PubMed PMID: 33320883; PubMed Central PMCID: PMCPMC7737895 following competing interests: GT and $L X$ are the technical directors of the department of R\&D in Yaneng Biotech, Co., Ltd, and receive research funding from this company. WD, JG, GL, NL, JP, CC, HD, JY, XZ, HH. are employees of Yaneng Biotech, Co., Ltd. 
16. Broughton JP, Deng X, Yu G, Fasching CL, Servellita V, Singh J, et al. CRISPR-Cas12-based detection of SARS-CoV-2. Nat Biotechnol. 2020;38(7):870-4. Epub 2020/04/18. doi: 10.1038/s41587-0200513-4. PubMed PMID: 32300245.

17. Kleter B, van Doorn LJ, ter Schegget J, Schrauwen L, van Krimpen K, Burger M, et al. Novel shortfragment PCR assay for highly sensitive broad-spectrum detection of anogenital human papillomaviruses. Am J Pathol. 1998;153(6):1731-9. Epub 1998/12/10. doi: 10.1016/S00029440(10)65688-X. PubMed PMID: 9846964; PubMed Central PMCID: PMCPMC1866345.

18. Gootenberg JS, Abudayyeh OO, Kellner MJ, Joung J, Collins JJ, Zhang F. Multiplexed and portable nucleic acid detection platform with Cas13, Cas12a, and Csm6. Science. 2018;360(6387):439-44. Epub 2018/02/17. doi: 10.1126/science.aaq0179. PubMed PMID: 29449508; PubMed Central PMCID: PMCPMC5961727.

19. Crannell Z, Castellanos-Gonzalez A, Nair G, Mejia R, White AC, Richards-Kortum R. Multiplexed Recombinase Polymerase Amplification Assay To Detect Intestinal Protozoa. Anal Chem. 2016;88(3):1610-6. Epub 2015/12/17. doi: 10.1021/acs.analchem.5b03267. PubMed PMID: 26669715.

20. Wang B, Wang R, Wang D, Wu J, Li J, Wang J, et al. Cas12aVDet: A CRISPR/Cas12a-Based Platform for Rapid and Visual Nucleic Acid Detection. Anal Chem. 2019;91(19):12156-61. Epub 2019/08/29. doi: 10.1021/acs.analchem.9b01526. PubMed PMID: 31460749.

\section{Supporting Information}

Materials and methods

Figure S1. Detection of 13 types of HR-HPVs using conventinal RPA primer pool

Figure S2. Evaluation of SPF primer pool

Figure S3. Evaluation of the GP5/GP6 primer pool

Figure S4. Primer titration for the detection of HPV16

Figure S5. Evaluation of the RPA-only method for HPV 16 detection

Figure S6. Evaluation of the Cas12a-only method for HPV 16 detection

Table S1. Conventional RPA primer pool

Table S2. SPF primer pool

Table S3. Enhanced PGMY/GP6+ primer pool

Table S4. crRNA pool and reporter used in this study 
Figures

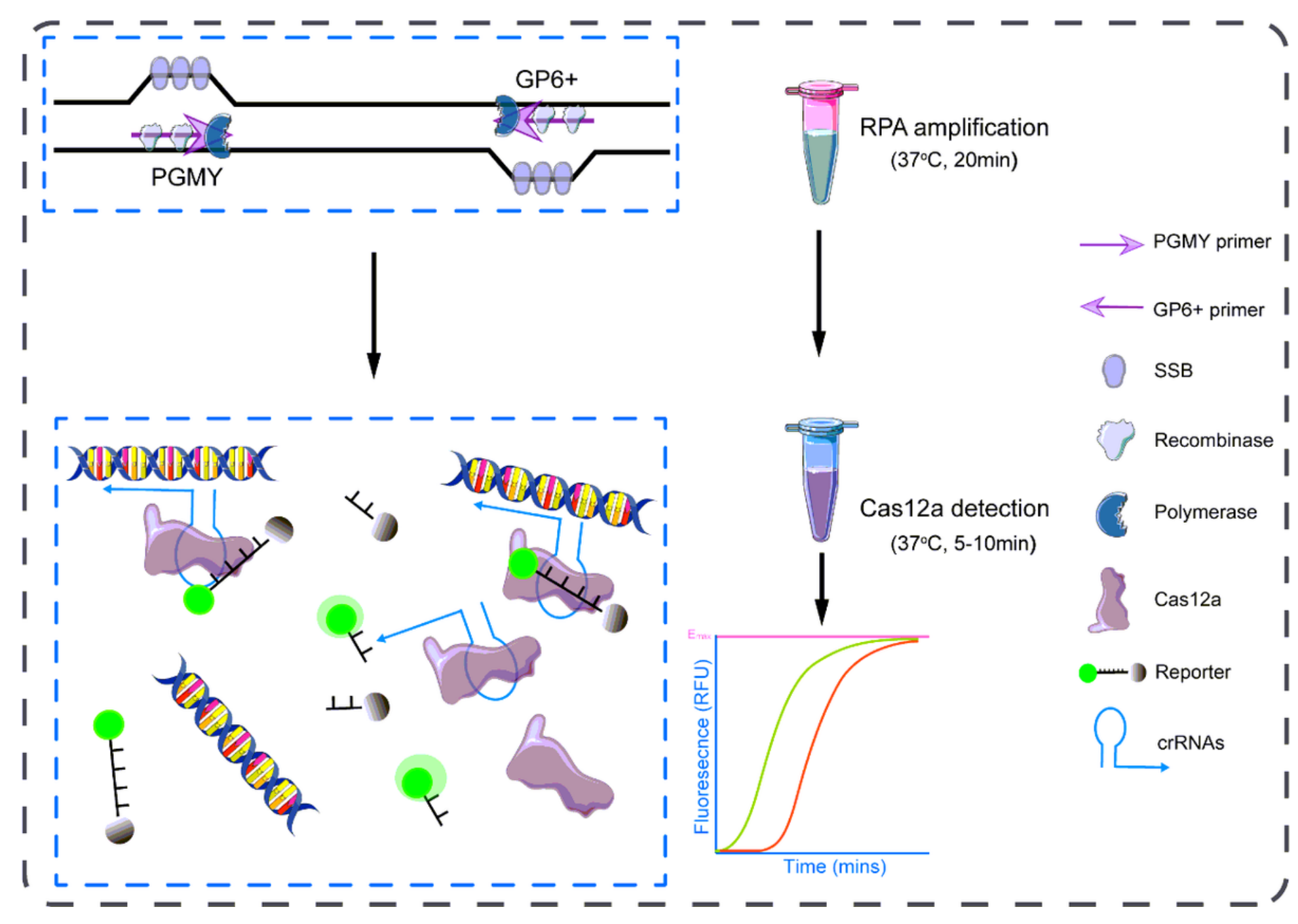

Figure 1

Illustration of the RPA-Cas12a method for HR-HPVs detection The system includes an RPA amplification with a pool derived from PGMY/GP6+ primer set that is able to amplify 13 types of HR-HPV in a reaction, and a Cas12a detection with a crRNA pool. The results can be achieved within 30 minutes using a fluorescent reader. 


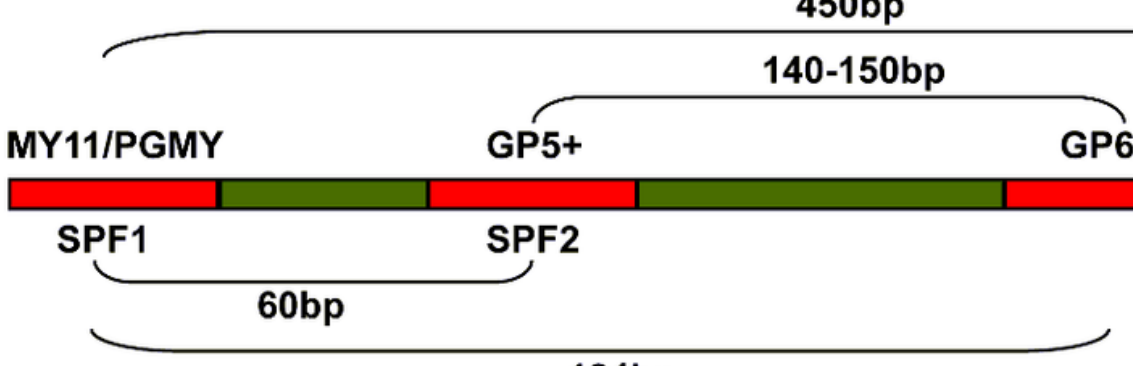

\section{4bp}

Conservative $L 1$ region

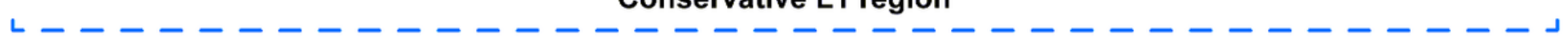

\section{B}

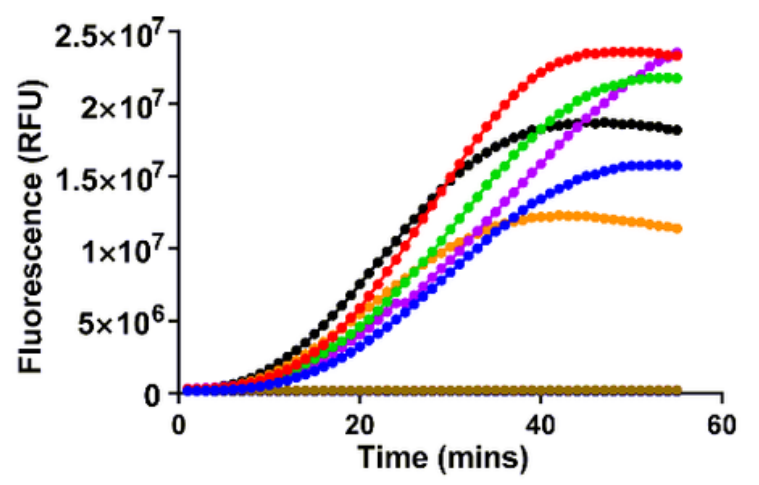

D

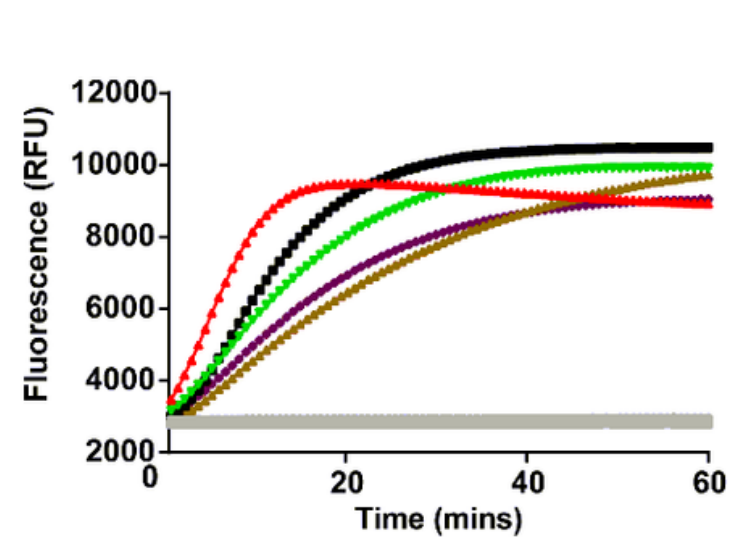

- H16-100

$\rightarrow \mathrm{H} 16-1000$

$\rightarrow \mathrm{H} 16-10000$

$\rightarrow \mathrm{H} 18-100$

$\rightarrow \mathrm{H} 18-1000$

$\rightarrow \mathrm{H} 18-10000$

$\rightarrow$ H16-NTC

$\rightarrow$ H18-NTC

\section{Figure 2}

\section{C}

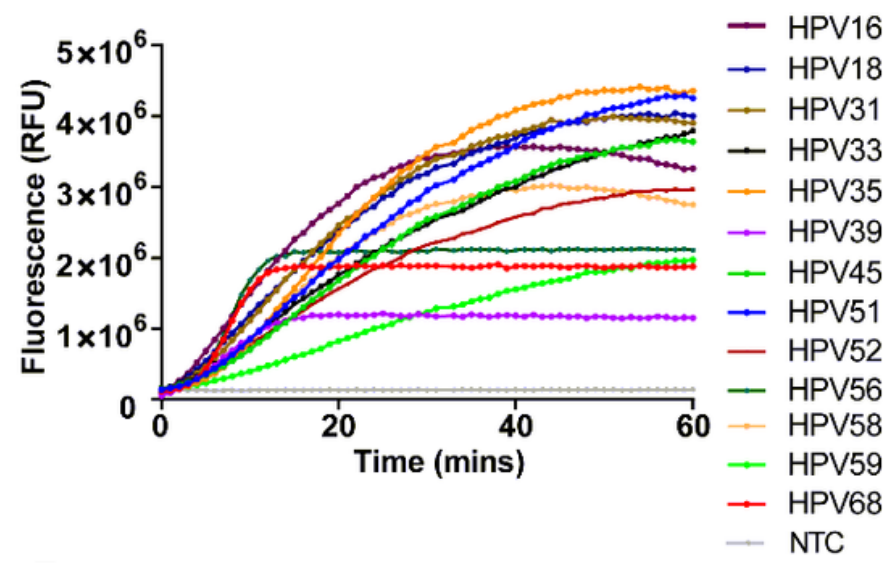

E

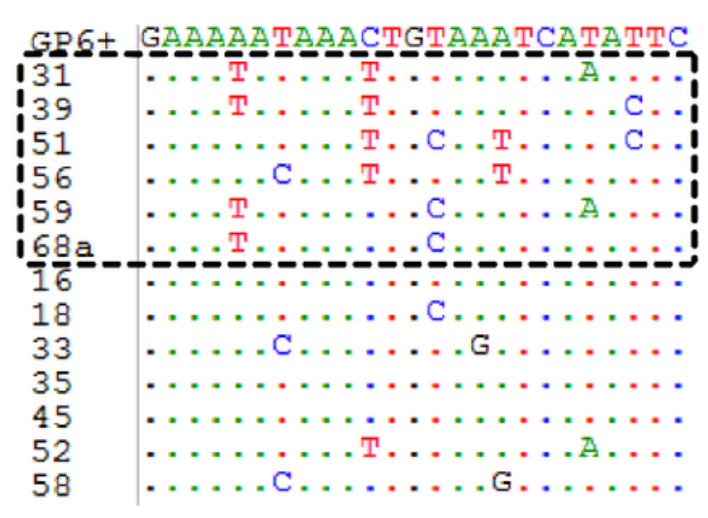

Evaluation of the PGMY/GP6+ primer pool (A) Schematic showing the relative position of the primer sets locating in the conservative L1 region of HPV. (B) Plasmids with different copy numbers of HPV16 and HPV18 were amplified by RPA reaction using the PGMY/GP6+ primer pool. NTC, no-template control. (C) Real-time fluorescence detection of HPVs with the crRNA pool containinig $11 \mathrm{crRNAs}$. For a single reaction, $5 \mu \mathrm{g}$ of each individual HPV plasmid was used. NTC, no-template control. (D) The PGMY/GP6+ pool was tested using 10000 copies of the indicated HPV plasmids for each RPA reaction. The RPA reaction was performed at $37^{\circ} \mathrm{C}$ for $20 \mathrm{~min}$ and followed by Cas $12 \mathrm{a}$ detection at $37^{\circ} \mathrm{C}$ for up to 60 minutes. NTC, no-template control. (E) Nucleotide sequence alignments of GP6+ (positions 5879 to 5903 according to HPV16 sequence, Genbank accession number NC_001526.4) to 13 HR-HPV genotypes. HPV 
genotypes are identified by numbers on the left. Dots indicate the presence of nucleotides identical to the top sequence (GP6+). HPV Types with weak amplification by the primer pool are shown by boxes.

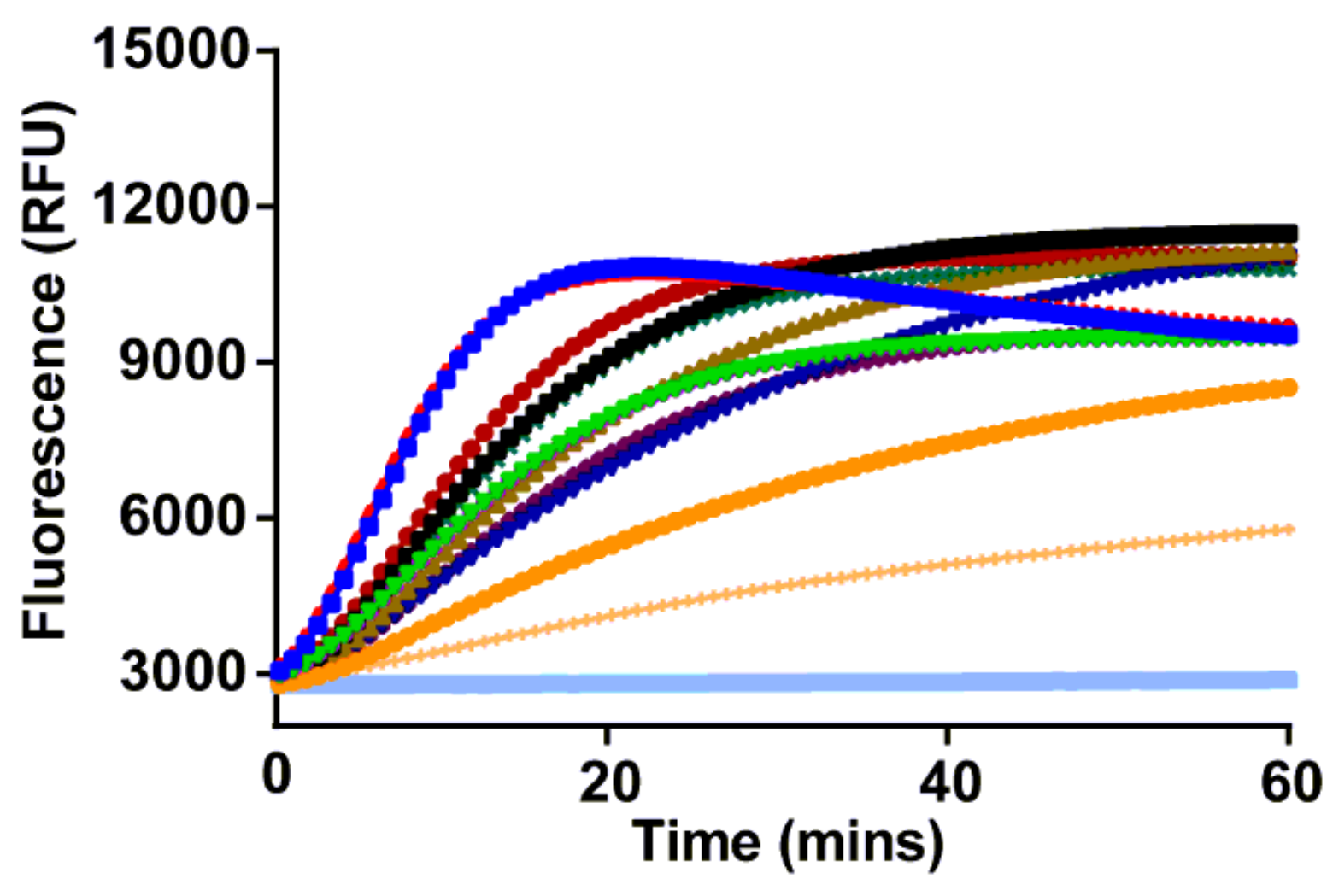

$\rightarrow$ HPV16

$\rightarrow$ HPV18

$\rightarrow$ HPV31

$\rightarrow$ HPV33

$\multimap$ HPV35

$\rightarrow$ HPV39

$\rightarrow$ HPV45

$\rightarrow$ HPV51

$\rightarrow$ HPV52

$\rightarrow$ HPV56

$\rightarrow$ HPV58

$\rightarrow$ HPV59

- HPV68

$\rightarrow$ NTC

Figure 3

Evaluation of the enhanced PGMY/GP6+ primer pool The PGMY/GP6+ primer pool was supplemneted with additional four primers that have less mismatches to HPV31, 39, 51, 56, 59, 68. The new pool was tested using 10000 copies of plasmid for each RPA reaction. NTC, no-template control. 

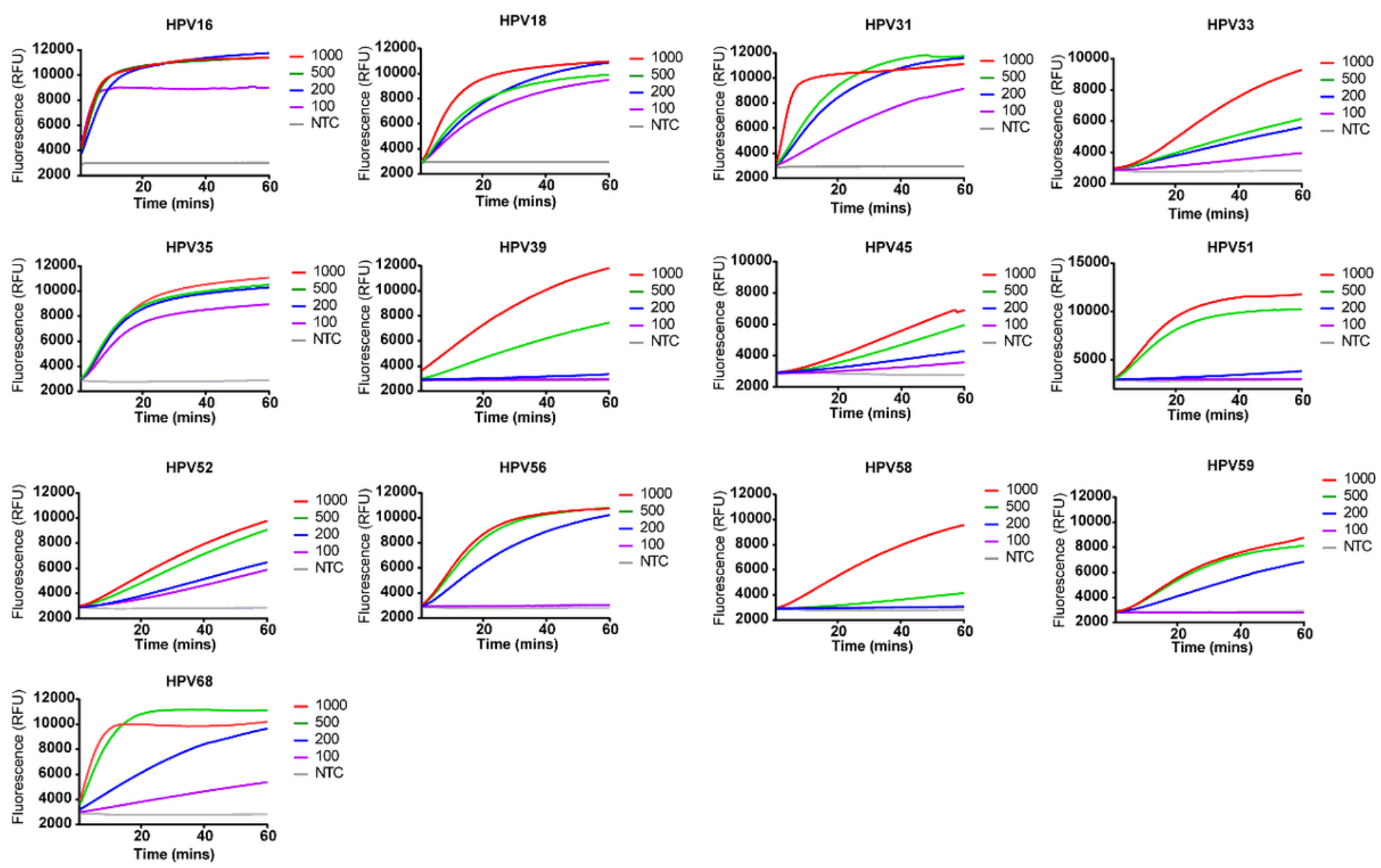

\section{Figure 4}

Serial dilutions of the indicated HPV plasmid for LoD determination. After RPA amplification at $37^{\circ} \mathrm{C}$ for 20 minutes using 10000 copies of plasmid for each reaction, $10 \mu \mathrm{L}$ of the yeild was transferred to $40 \mu \mathrm{L}$ of the Cas12a mixture for cleavage assays. NTC, no-template control.

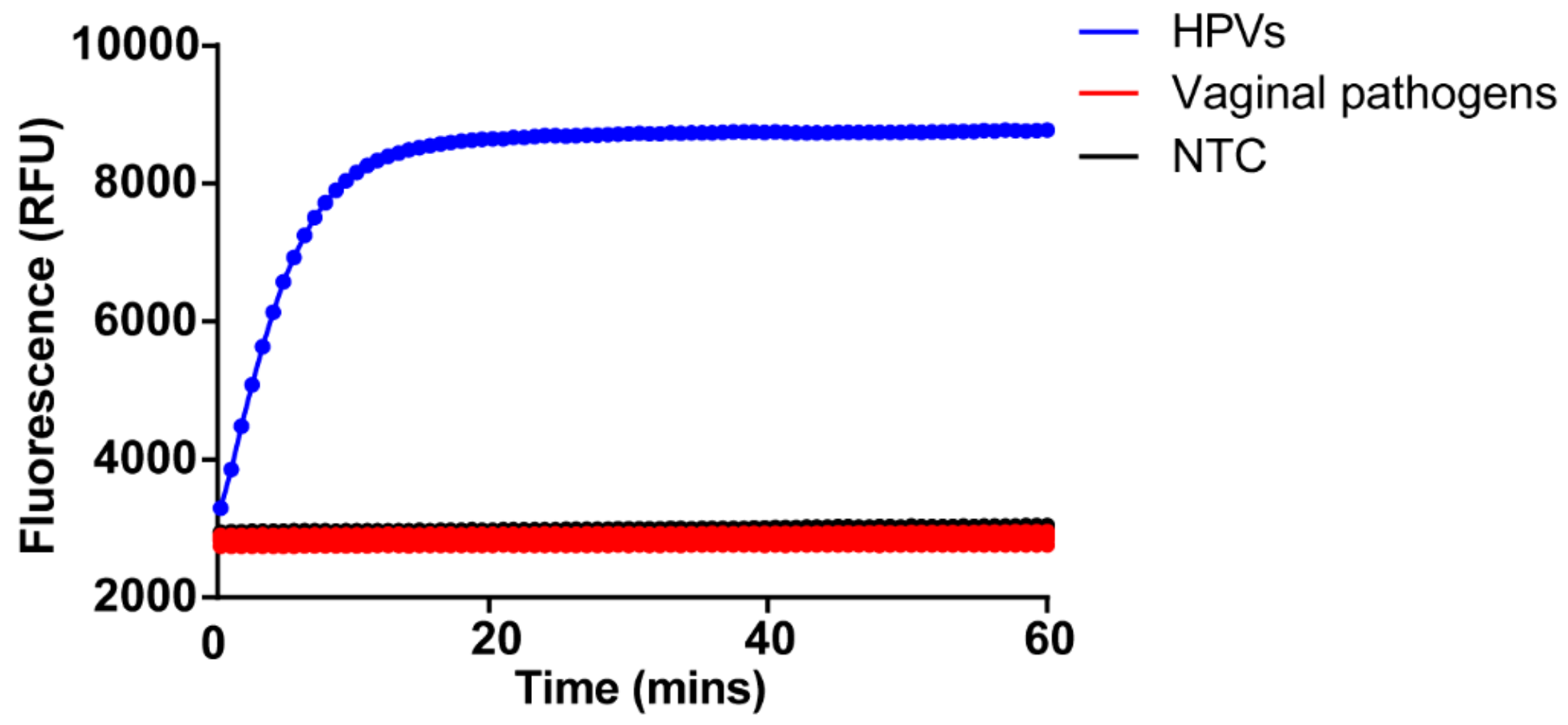




\section{Figure 5}

Specificity assessment of the RPA-CRISPR assay for HPVs. Only the DNA from HPVs produced signals, whereas plasmids from other pathogens and the negative control did not produce any detected signals. The positive control was made by amplifying the HPV mixture where a total of $13 \mathrm{HR}-\mathrm{HPV}$ s were mixed at the concentration of 10000 copies/ $\mu \mathrm{L}$ for each type. NTC, no-template control.
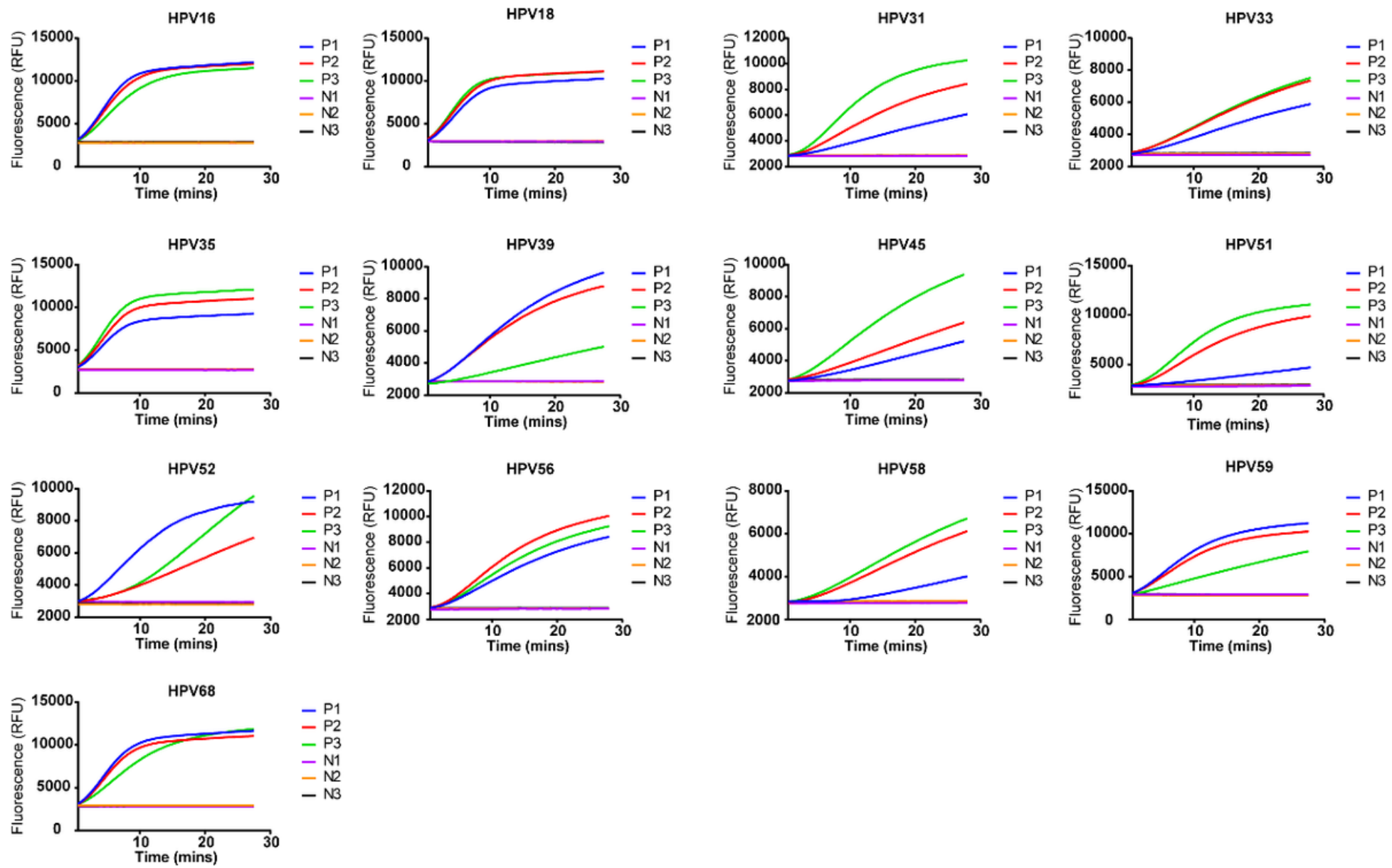

Figure 6

Three positive and three negative samples identified by qPCR were used for testing for each type. The developed assay had $100 \%$ positive and negative agreements relative to the GPCR assay, for detection of the 13 types of HR-HPVs. P, positive; N, negative.

\section{Supplementary Files}

This is a list of supplementary files associated with this preprint. Click to download.

- Supportinginformation.docx 